Liina Lukas

\title{
IX. INTERNATIONALES SYMPOSIUM ZUR BALTISCHEN LITERARISCHEN KULTUR: MEDIEN DER AUFKLÄRUNG. AUFKLÄRUNG DER MEDIEN
}

Im Rahmen der estnisch-deutschen akademischen Woche Academica fand am Anfang September in Tartu das IX. Symposium zur baltischen literarischen Kultur statt, das diesmal die "Medien der Aufklärung" und zugleich eine "Aufklärung der Medien“ zum Thema hatte. Die Symposien zur baltischen literarischen Kultur werden seit 2001 zur Erforschung der baltischen Kulturgeschichte in einer Fächer-, Länder- und Wissenschaftstraditionen übergreifenden Zusammenarbeit abgehalten. Das diesjährige Symposium widmete sich der Wissensvermittlung im Zeitalter der Aufklärung aus der Perspektive verschiedener Fächer wie der Geschichtswissenschaft, Literaturwissenschaft, Philosophie, Kunstgeschichte, Folkloristik, Theologie und der Politologie.

Die Wurzeln der heutigen Informationsgesellschaft liegen im Zeitalter der Aufklärung. Just um diese Zeit entstand die Medienlandschaft bzw. diese Form von Öffentlichkeit, wie wir sie heute kennen. Das Wissen wurde zum Grundstein einer neuen 


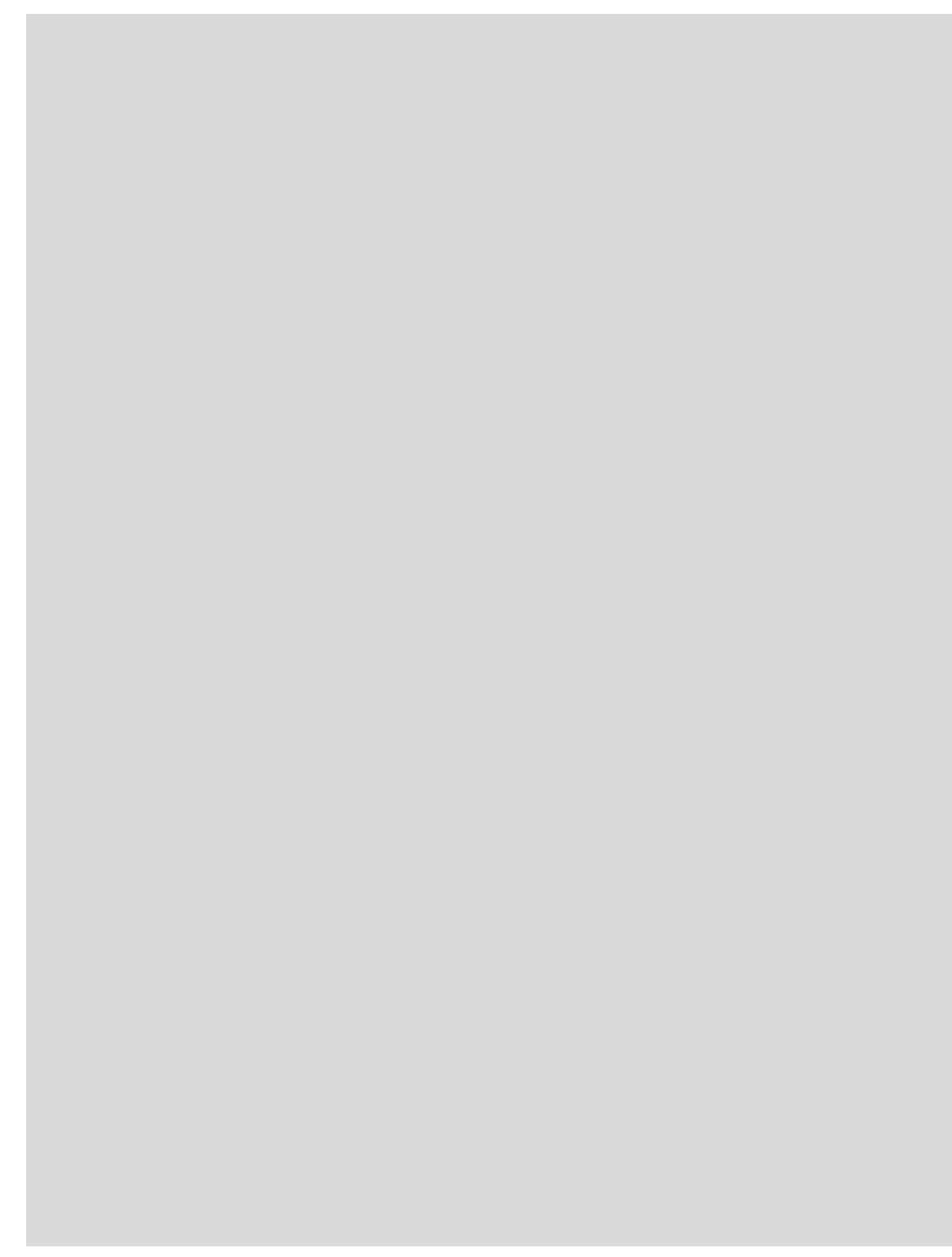

Abb. 1. Plakat der Sommerschule. Illustration zum Garlieb Merkels Werk „Die Vorzeit Livlands" (1798), welche den Kampf Laokoons mit der Schlange darstellt.

Gesellschaftsordnung. Erwerb, Vermittlung und Vertrieb von Wissen, zunächst nur ein Privileg der elitären respublica litteraria, schloss im Zuge der Volksaufklärung endlich alle Gesellschaftsschichten mit ein. Auch unter Bauern reichte die traditionelle, unmittelbare mündliche
Weitergabe von Wissen nicht mehr aus. Die Wissensgesellschaft setzte Lesefähigkeit voraus. Das Lesen und der anschließende Gedankenaustausch über das Gelesene wurden zu einer neuen Form der Kommunikation, welche die bisherigen Arten der Wissensvermittlung verdrängte und den Aufstieg neuer medialer Formen wie Enzyklopädien oder Zeitschriften sowie des Romans mit sich brachte.

Auch im Baltikum fanden diese Transformationsprozesse statt. Auch dort entstanden neue Arten von Kommunikationsnetzwerken Freimaurerlogen, Klubs, Lesezirkel, Bibliotheken, Zeitungen, Theater sowie generell eine literarische Öffentlichkeit. Die Ostseeprovinzen des Zarenreiches gehörten zum Kommunikationsraum der europäischen Aufklärung. Gleichwohl war die baltische Aufklärung in besonderer Weise eine "geteilte Aufklärung", weil sie mehr als anderswo in Europa mit dem Kolonialismus und mit der Problematik der ethnischen Dominanz verbunden war. Die horizontale, deutschsprachige, gelehrte Aufklärung kreuzte sich hier mit der vertikal ausgerichteten Volksaufklärung, deren Hauptträger deutschsprachige Gelehrte und Gebildete (vor allem Pastoren) und deren Hauptadressaten die "Undeutschen“, d. h. Esten und Letten, waren. Da hier die sozialen und sprachlichen Grenzen übereinstimmten, bedingte die Sprachwahl zugleich besondere Medien und Adressatenmodelle.

Wie haben die neuen Ideen und Ideale aus den Zentren der europäischen Aufklärung die baltischen Länder erreicht und welche Form haben sie aufgrund der kolonialen Situation angenommen? Welches Verhältnis hatte die europäische Aufklärung generell zum Kolonialismus? Wo entstanden Zentren und was waren wichtige Kommunikationswege (sowohl in den baltischen Ländern als auch anderswo in Europa)? Inwiefern gab es einen intensiven Gedankenaustausch mit den Zentren der Aufklärung? Wer waren die wichtigsten Vertreter der baltischen Aufklärung und an wen richtete sich die Aufklärung? Welche Funktionen hatten die unterschiedlichen Sprachen im Vermittlungsprozess? Inwiefern ergänzt oder erweitert die baltische Aufklärung das Gesamtbild der europäischen Aufklärung? Welche "geteilten“ Erfahrungen und Formen gilt es noch in der europäischen Aufklärung zu entdecken? Was sind die Sprachen der europäischen Aufklärung und inwieweit sind die Medienpraktiken durch die Sprache bedingt? 


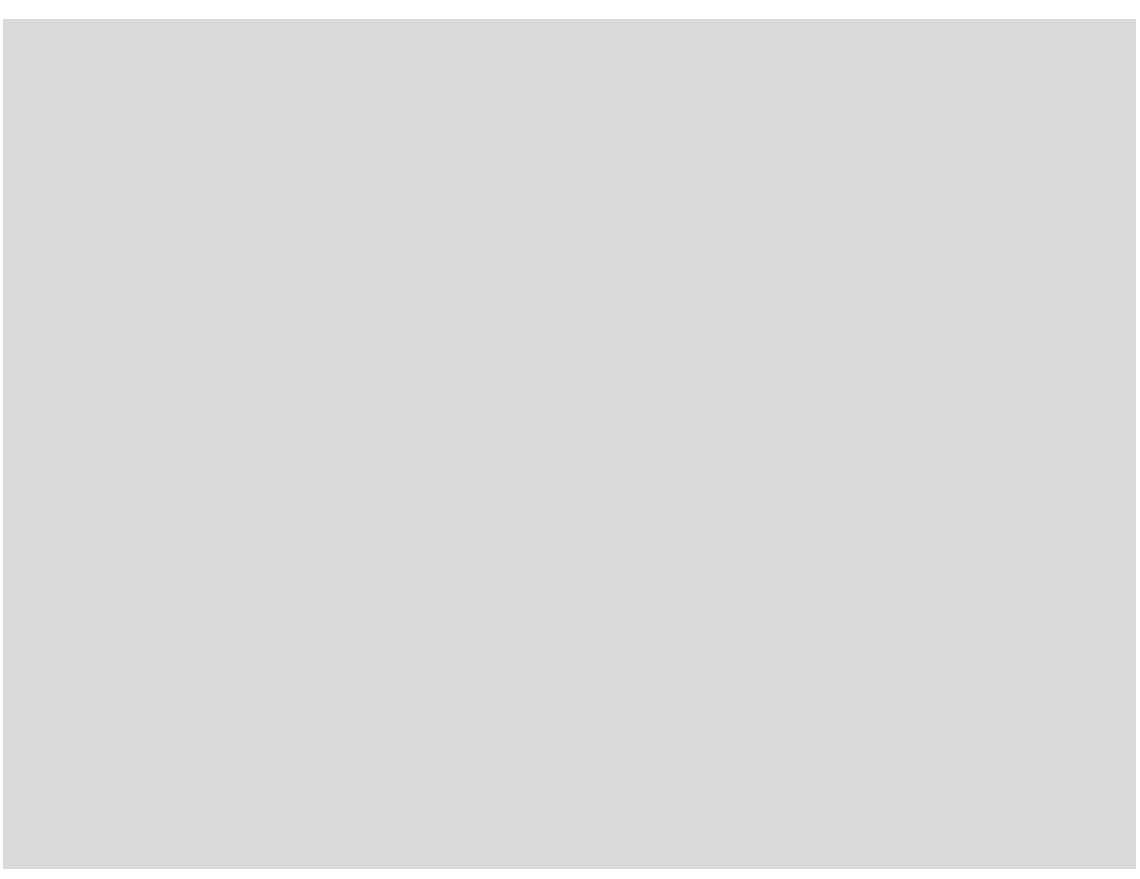

Abb. 2. Sommerschule in Riga (2017). Vor dem Denkmal Herders. Foto: Tiina-Erika Friedenthal.

Das waren die Kernfragen, über die die Wissenschaftler aus Deutschland, Lettland, Frankreich und Estland drei Tage diskutiert haben.

Das Symposium fand parallel zur internationalen Sommerschule „Die baltische Aufklärung und ihr Erbe“ (28.08.-09.09.2017, siehe auch https://sisu.ut.ee/mdad in Riga und Tartu statt, im Laufe dessen die Schlüsselthemen der baltischen Aufklärung behandelt wurden: das Volkslied und seine Bedeutung für die Entwicklung der kleinen Kulturen; die Volksaufklärung unter kolonialen Bedingungen und Garlieb Merkels „Letten“ als Schlüsseltext der späteren Historiographie; Naturverständnis und Ökologie der baltischen Aufklärung; Frauen und Aufklärung, Bühne und Kanzel als Katheder der Aufklärung, theologische Perspektiven der Aufklärung, Patriotismus versus Kosmopolitismus der Aufklärung in der Diskussion zwischen Kant und Herder, die Universitätsgründung in Tartu einem aufklärerischen Bildungsprogramm folgend, das Erbe der Aufklärung am Beispiel der Romane von Jaan Kross. Die
Sommerschule fand ihren Höhepunkt in der Inszenierung der Komödie „Hofmeister“ des Dorpater (Tartuer) Dichter Jakob Michael Reinhold Lenz. In der Sommerschule erfolgten Workshops zu Digital Humanities, die nach den digitalen Mitteln zur Erforschung der Aufklärung suchten und diese auch erprobten.

Das Symposium und die Sommerschule fanden im Rahmen des Projekts „Medienpraktiken der Aufklärung“ statt, das in der Erasmus+ Programmlinie "Strategische Hochschulpartnerschaften" in Zusammenarbeit der Universitäten Tartu, Potsdam, Riga und Bordeaux-Montaigne organisiert wird.

\section{AUF SPUREN DER AUFKLÄRUNG IN DORPAT}

Johann Gottfried Herder beginnt sein „Journal meiner Reise im Jahr 1769“ mit folgenden Worte: „Den 23 Mai/3 Jun. reisete ich aus Riga ab und den 25/5. ging ich in See, um ich weiß nicht wohin? zu gehen. Ein großer Theil unsrer Lebensbegebenheiten hängt würklich vom Wurf von Zufällen ab. So kam ich nach Riga, so in mein geistliches Amt und so ward ich deßelben los; so ging ich auf Reisen."

Stellen wir uns kurz vor, es wäre das Jahr 1769 - das Jahr, in dem Johann Gottfried Herder von Riga Abschied nahm. Er würde aus der livländischen Metropole Riga nach Dorpat kommen, in die zweitgrößte Stadt Livlands - nach Tartu, wie wir Esten diese Stadt nennen und auch damals nannten. Was würde er hier vorfinden? Wen würde er hier treffen?

Im Vergleich zu Riga, dem Zentrum aufklärerischen Denkens, findet er hier eine Situation vor, die aufklärerischen Idealen und Ansprüchen wenig Genüge leistet. Erst etwa 15 Jahre später, 1785, wird in dem verträumten Städtchen eine Buchhandlung eröffnet, die ein Jahr später zu einer Lesebibliothek erweitert wird. 1769 sind diejenigen, die Lesehunger verspüren, auf die Buchhandlung Johann Friedrich Hartknochs in Riga angewiesen.

Eine regelmäßig erscheinende Zeitung wird erst mit der Eröffnung einer stadteigenen Druckerei ab 1789 erscheinen: die von Friedrich Gotthilf Findeisen, Friedrich David Lenz und Christian Heinrich Nielsen gegründete Dörptsche Politische Gelehrte Zeitung wird ein 9/2 (Frankfurt: Deutscher Klassiker Verlag, 1997), 9-37, hier 11. 
Jahr später vom Buchdrucker M. G. Grenzius übernommen und als Dörptsche Zeitung herausgegeben. Lesegesellschaften und Klubs (damals Klubben genannt), in denen Gedankenaustausch stattfinden kann, werden erst in den neunziger Jahren des 18. Jahrhunderts gegründet.

Dorpat, das spätere „Embach-Athen“, hat im Jahre 1769 keinen Hartknoch, keinen Hamann und auch keinen Merkel. Aber so ganz "dunkel“ ist es hier auch im Jahre 1769 wohl nicht. Herder besucht selbstverständlich Friedrich Konrad Gadebusch, welcher der geistige Mittelpunkt Dorpats ist und mit seinen „Livländischen Jahrbüchern“, seinen "Abhandlungen von livländischen Geschichtsschreibern“ sowie mit seiner Zeitschrift „Versuche in der livländischen Geschichtskunde und Rechtsgelehrsamkeit" nicht nur der wichtigste Sammler und Enzyklopädist, sondern auch der Begründer der wissenschaftlichen Geschichtsforschung in Livland (sprich im Baltikum) und einen Wegbereiter einer „modernen“ Geschichtswissenschaft darstellt. ${ }^{2}$

Herder trifft hier wohl auch einen Studiengefährten aus Königsberg, Friedrich David Lenz, mit dem er im Jahre 1762 in Königsberg immatrikuliert worden war, und dessen Bruder, den noch blutjungen Jakob Michael Reinhold Lenz, der unter dem geistigen Einfluss von Gadebusch und dessen recht reichhaltiger Bibliothek - im Pfarrhaus der Johanniskirche (Jaani kirik, der damals einzigen lutherischen Kirche in Dorpat) aufgewachsen ist. Im Jahre 1769 ist Jakob schon Dichter, und nicht nur in seinen eigenen Augen. Ein Gedicht von ihm ist im Jahr 1766 in den „Rigischen Anzeigen“ veröffentlicht worden, mit einem Vorwort von Theodor Oldekop, dem Pastor der estnischen Gemeinde der Johanniskirche, der dem erst 15-jährigen Sohn des Oberpastors eine glänzende Dichterlaufbahn vorausgesagt hat: „Ein solches seltenes Genie verdient alle Aufmunterung. Ich hoffe, die Leser werden mit mir wünschen, dass die dichterischen Gaben dieses Hofnungsvollen Jünglings sich immer mehr zur Ehre unsers Vaterlandes entwickeln und erhöhen mögen“. ${ }^{3}$

Lenz hat auch schon sein erstes Drama geschrieben, den "Verwundeten Bräutigam", das im benachbarten Oberpahlen (Põltsamaa) zu einer Adelshochzeit aufgeführt worden ist (oder

2 Siehe Chistina Kupffer, Geschichte als Gedächtnis. Der livländische Historiker und Jurist Friedrich Konrad Gadebusch (1719-1788) (Köln: Böhlau, 2004).

3 Theodor Oldekop, ,,An den Leser“, Gelehrte Beiträge zu den Rigischen Anzeigen auf das Jahr 1766, VII. Stück (Riga, 1761-1767), 49. eben so gut wie aufgeführt worden wäre). ${ }^{4}$ Im Jahre 1769 studiert er in Königsberg und hat eben sein erstes Buch herausgegeben „Die Landplagen" - ein Epos in Hexametern, das er der russischen Kaiserin Katharina II. gewidmet hat.

Jakob Lenz ist aber nur kurz hier in Dorpat, ungefähr wie „ein vorübergehender Meteor", könnte man sagen, um seinen Vater zu besuchen, den er noch von Königsberg aus in einem Brief darum gebeten hat, ein teuer gebundenes Exemplar des Buches der Kaiserin zu übersenden. Der Vater hat das allerdings nicht getan ... ${ }^{5}$

Wenn man sich heutzutage an den Ufern des Embachs (Emajõgi) auf Spuren der Aufklärung macht und nach entsprechenden "Erinnerungsorten“ (Mnemotopen) umsieht, ist die Johanniskirche wohl die erste Adresse - das wichtigste geistige Zentrum der alten Stadt, einer der wenigen Orte, die die großen Feuersbrünste der Jahre 1755, 1763 und 1775 überstanden hat. Bis zur Wiedereröffnung der Universität im Jahre 1802 gilt in Dorpat die Kanzel als Katheder der Aufklärung.

Der eigentliche Katheder der Aufklärung entsteht erst mit der Wiedereröffnung der Universität, die Dorpat zur geistigen Hauptstadt der Ostseeprovinzen Russlands, dem eigentlichen Vermittlungspunkt des Wissens und dem Treffpunkt vieler Nationen macht. Wenn überhaupt irgendwo im Baltikum, dann gerade hier trafen sich Deutsche, Esten, Letten, Russen und dazu noch viele Zugereiste wie beispielsweise der erste Rektor Georg Friedrich Parrot, der französische Wurzeln hat, oder Karl Morgenstern, der aus Deutschland kommt, um Professor für klassische Philologie und Ästhetik zu werden und mit seiner Privatbibliothek von 12.000 Bänden den Grundstock zur Universitätsbibliothek zu legen. Er, Morgenstern, ist es, der im Jahre 1805 auf einer Auktion der Herderschen Bibliothek in Weimar diese Büchersammlung für die Universitätsbibliothek Dorpat erwirbt (von 500 Büchern sind allerdings wegen eines Schiffbruchs

4 So meint Heinrich Bosse in seiner kürzlich erschienenen Lenz-Biographie: „Nach Blums Bericht war das Drama als ,Festspiel' gedichtet und zur Hochzeit aufgeführt worden, eine hrsg. von Julia Freytag, Inge Stephan, Hans-Gerd Winter (Berlin, Boston: DeGruyter, 2017), 8-9. 5 So Bosse, „Leben“, 12: „Es ist nicht anzunehmen, dass der Vater die zweifelhafte Dichtung seines Sohnes nach Petersburg weiterbeförderte. Es ist vielmehr anzunehmen, dass er ihn durch Vorwürfe zusatzlich beschämte, zumal Hehn in seinem Brief angedeutet hatte, Jakob Lenz werde sich in literarische Auseinandersetzungen einlassen und damit seine theologische Ausbildung aufs Spiel setzen." 
nur 170 eingetroffen $\left.{ }^{6}\right)$. Oder Friedrich Maximilian Klinger, der aus Deutschland über St. Petersburg nach Dorpat kommt, um Kurator der Universität zu werden. Während Klinger in der deutschen Kulturgeschichte als Namensgeber des literarischen „Sturm und Drang" bekannt ist, wird er in Dorpat mit seinem Theaterverbot für mehr als 60 Jahren (von 1803 bis 1867) eine allerdings rätselhafte, vieldeutige Spur hinterlassen.

Die Gründung der neuen Universität - der Kaiserlichen Universität zu Dorpat, die die einzige deutschsprachige Universität im ganzen „Reich der Reußen“ war, mit der einzigen lutherischen theologischen Fakultät, bildete das Großereignis der Aufklärung in dieser Region. Sie fußt auf einem aufklärerischen Bildungsprogramm: „Es ward Licht! “7 stellt der frischgebackene Rektor Parrot in seiner Rede zur Eröffnung der Universität 1802 schlicht und einfach fest. Er hegt die Hoffnung, mit dieser Universitätsgründung „etwas wichtiges zum Besten der Aufklärung zu leisten“. Und er hat recht gehabt, denn es wurde Licht. Die Gründungstat erfolge nämlich „aus ächter Liebe für die Aufklärung“, soll „den Fortschritten des menschlichen Geistes neue Bahn eröffnen“ und getragen sein von den Werten „Liberalität, Humanität und wahre Würde". ${ }^{8}$

Diese aufklärerische Werte haben an der heutigen Universität Tartu nichts von ihrer Bedeutung eingebüßt.

\footnotetext{
6 Malle Ermel, „Kulturerbe im digitalen Zeitalter. Ubersicht über die Aktivitäten de Universitätsbibliothek Tartu“, Bibliothek der Zukunft. Zukunft der Bibliothek: Festschrift fü Elmar Mittler, hrsg. von Andreas Degkwitz (Berlin, Boston: DeGruyter, 2016), 34-44, hier 35

7 Friedrich George Parrot, ,,Rede ueber einige Ansichten der Naturkenntnisse, in Ansehung ihres Einflusses auf Menschen-Cultur“, Geschichte und Beschreibung der Feyerlichkeiten bey Gelegenheit der am 21sten und 22sten April 1802 geschehenen Eröfnung der neu angelegten Kayserlichen Universität zu Dorpat in Lievland, hrsg. von Gottlob Benjamin Jäsche (Dorpat: M. G. Grenzius, 1802), 46.

8 Ibidem, 43.
}

Liina Lukas: IX International Symposium on Baltic Literary Culture: Media of the Enlightenment. Enlightenment of the Media

Keywords: The Baltic Enlightenment; The Social Networks in the Age of Enlightenment; Johann Gottfried Herder; Garlieb Merkel; Popular Enlightenment; Tartu in the $18^{\mathrm{TH}}$ Century

\section{CV}

Liina Lukas (b. 1970) is Associate Professor for Comparative Literature at the Institute of Cultural Research at the University of Tartu. Her research interests are Estonian-German literary contacts, BalticGerman literature and early Estonian literature. 
